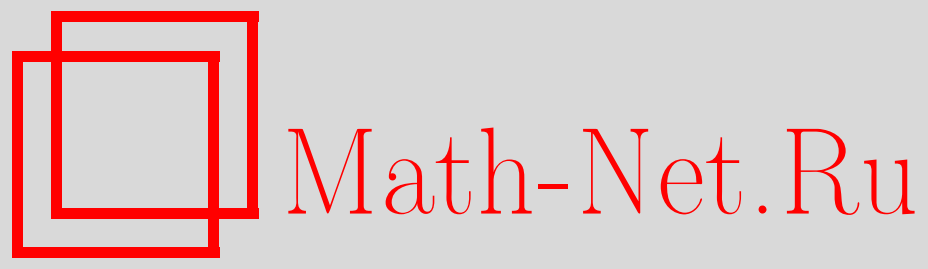

А. В. Покровский, Устранимые особенности решений линейных равномерно эллиптических уравнений второго порядка, Функи. анализ и его прил., 2008, том 42, выпуск 2, 44-55

DOI: https://doi.org/10.4213/faa2901

Использование Общероссийского математического портала MathNet.Ru подразумевает, что вы прочитали и согласны с пользовательским соглашением http://www . mathnet.ru/rus/agreement

Параметры загрузки:

IP: 34.239 .49 .27

26 апреля 2023 г., 13:11:04

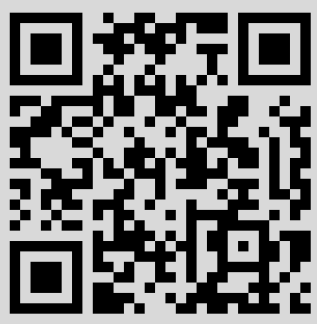




\title{
Устранимые особенности решений линейных равномерно эллиптических уравнений второго порядка
}

\author{
(c) 2008. А. В. Покровский
}

\section{Введение}

Пусть $G$ - ограниченная область в $\mathbb{R}^{n}(n \geqslant 2)$ и

$$
L f=\sum_{i, j=1}^{n} D_{i}\left(a_{i j}(x) D_{j} f\right), \quad x=\left(x_{1}, \ldots, x_{n}\right) \in G, D_{i}=\frac{\partial}{\partial x_{i}},
$$

- равномерно эллиптический линейный дифференциальный оператор второго порядка с ограниченными и измеримыми действительными коэффициентами $a_{i j}(x)=a_{j i}(x)$ в $G(i, j=1, \ldots, n)$. Это означает, что существуют такие $\lambda, \Lambda>0$, что для всех $\xi=\left(\xi_{1}, \ldots, \xi_{n}\right) \in \mathbb{R}^{n}$ и для почти всех $x \in G$ выполняются неравенства

$$
\sum_{i, j=1}^{n} a_{i j}(x) \xi_{i} \xi_{j} \geqslant \lambda|\xi|^{2}:=\lambda\left(\xi_{1}^{2}+\cdots+\xi_{n}^{2}\right) \quad \text { и } \quad \sum_{i, j=1}^{n} a_{i j}^{2}(x) \leqslant \Lambda^{2} .
$$

Через $A_{L}(G)$ обозначим множество всех обобщенных решений уравнения $L f=0$ в $G$ (в дальнейшем мы рассматриваем только действительные функции, а обозначение $A_{L}(G)$ используется также и в случае, когда $G$-открытое множество, не обязательно являющееся областью). Напомним, что по теореме Де Джорджи и Нэша всякое обобщенное решение этого уравнения непрерывно и локально гёльдерово в $G$.

Пусть $H(G)$ - некоторый класс функций, определенных в области $G$. В дальнейшем всегда будем предполагать, что $A_{L}(G) \subset H(G) \subset C(G)$. Пусть $K-$ компакт в $G$. Мы будем называть $K$ устранимым для обобщенных решений уравнения $L f=0$ (или $L$-устранимым) в классе $H(G)$, если для любой функции $u \in H(G)$, сужение которой на $G \backslash K$ принадлежит $A_{L}(G \backslash K)$, существует такая функция $\bar{u} \in A_{L}(G)$, что $u \equiv \bar{u}$ на $G \backslash K$.

Если $x \in \mathbb{R}^{n}$ и $r>0$, то $B(x, r)$ и $S(x, r)$ обозначают соответственно открытый евклидов шар с центром в точке $x$ радиуса $r>0$ и его границу. Положим $G_{r}:=\{y \in G: B(y, r) \Subset G\}$.

Пусть $r>0, x \in G_{r} \neq \varnothing, f \in C(S(x, r))$, и пусть $f_{L, x, r}-$ непрерывная функция на замкнутом шаре $\bar{B}(x, r):=\left\{y \in \mathbb{R}^{n}:|x-y| \leqslant r\right\}$, такая, что ее сужение на $B(x, r)$ принадлежит $A_{L}(B(x, r))$ и $f_{L, x, r} \equiv f$ на $S(x, r)$. Такая функция всегда существует и единственна (см., например, [1, гл. 2, теорема 4.3]). Если $f \in C(G)$, то на множестве $G_{r}$ определим функцию $f_{L, r}(x):=f_{L, x, r}(x)$, которая, как устанавливается в приводимой ниже лемме 1 , будет непрерывной на $G_{r}$. 
Пусть $\alpha>0$. Мы говорим, что непрерывная в области $G$ функция $f$ принадлежит классу $U_{L}^{\alpha}(G)$, если существует такое $C \geqslant 0$, зависящее только от $f$, что для каждого шара $B(x, r) \Subset G$ норма функции $f-f_{L, x, r}$ в пространстве $C(B(x, r))$ не превосходит $C r^{\alpha}$, т. е. $\left\|f-f_{L, x, r} \mid C(B(x, r))\right\| \leqslant C r^{\alpha}$. Определим класс $C_{L}^{\alpha}(G)$ как множество всех функций $f \in C(G)$, для каждой из которых существует такое $C \geqslant 0$, зависящее только от $f$, что для любого шара $B(x, r) \Subset G$ имеет место неравенство $\left|f(x)-f_{L, r}(x)\right| \leqslant C r^{\alpha}$. Хаусдорфову меру компакта $K$ порядка $\alpha$ обозначим через $\operatorname{mes}^{\alpha} K$.

В принятых выше обозначениях сформулируем основной результат работы.

Теорема 1. Пусть $0<\alpha \leqslant 2$. Тогда для L-устранимости компакта $K$ в классе $U_{L}^{\alpha}(G)$ необходимо и достаточно, чтобъ выполнялось условие mes $^{n-2+\alpha} K=0$.

Замечание 1. Из приводимого ниже доказательства теоремы 1 и из включения $U_{L}^{\alpha}(G) \subset C_{L}^{\alpha}(G)$, непосредственно вытекающего из определений классов $U_{L}^{\alpha}(G)$ и $C_{L}^{\alpha}(G)$, следует, что теорема 1 остается справедливой, если в ее формулировке заменить класс $U_{L}^{\alpha}(G)$ на $C_{L}^{\alpha}(G)$.

Чтобы сформулировать следующий результат, введем такое обозначение: если $f \in C(\bar{B}(x, r))$, то оc $B(x, r) f:=\sup _{B(x, r)} f-\inf _{B(x, r)} f-$ колебание функции $f$ на шаре $B(x, r)$. Во введенных обозначениях упомянутая выше теорема Де Джорджи и Нэша может быть сформулирована следующим образом (см. [2, теорема 8.22 и замечание после теоремы 8.24]): для любой тройки концентрических шаров $B(x, r) \Subset B(x, R) \Subset B\left(x, R_{0}\right) \Subset G$ с $R \leqslant 1$ и для каждой функции $u \in A_{L}\left(B\left(x, R_{0}\right)\right)$ справедливо неравенство

$$
\underset{B(x, r)}{\operatorname{Osc}} u \leqslant C\left(\frac{r}{R}\right)^{\gamma} \underset{B(x, R)}{\operatorname{Osc}} u,
$$

где $C>0,0<\gamma \leqslant 1, C$ и $\gamma$ зависят только от $n$ и $\Lambda / \lambda$. Для заданного $\alpha \in(0,1)$

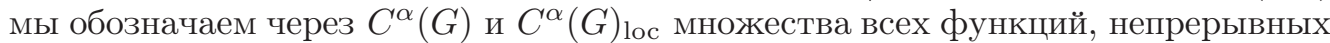
в $G$ и удовлетворяющих условию Гёльдера с показателем $\alpha$ соответственно в области $G$ и на ее компактных подмножествах. Аналогичным образом определяются классы $U_{L}^{\alpha}(G)_{\text {loc }}, C_{L}^{\alpha}(G)_{\text {loc }}, C^{1, \alpha}(G)_{\text {loc }}, Z(G)_{\text {loc }}, W_{p}^{1}(G)_{\text {loc }}$, где, как обычно, $C^{1, \alpha}(G)$ - множество всех функций $f$, непрерывно дифференцируемых в $G$, первые частные производные которых принадлежат $C^{\alpha}(G), Z(G)$ - множество всех функций $f \in C(G)$, удовлетворяющих условию Зигмунда $|f(x-h)-2 f(x)+f(x+h)| \leqslant C|h|$ для любых $[x-h, x+h] \subset G$ (с не зависящей от $x \in G$ и $h \in \mathbb{R}^{n}$ величиной $\left.C \geqslant 0\right)$, и $W_{p}^{1}(G)$ - пространство Соболева функций с суммируемыми в степени $p \in\{1,2\}$ первыми обобщенными производными в $G$. Из принципа максимума для обобщенных решений уравнения $L f=0$ следует, что при всех $\alpha \in(0,1)$ справедливо включение $C^{\alpha}(G)_{\text {loc }} \subset U_{L}^{\alpha}(G)_{\text {loc }}$. Следующая теорема показывает, что при достаточно малых $\alpha$ имеет место сов-

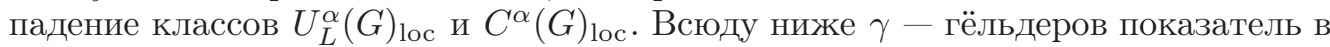
приведенной выше формулировке теоремы Де Джорджи и Нэша.

Теорема 2. Пусть $0<\alpha<\gamma$. Тогда классъц функиий $U_{L}^{\alpha}(G)_{\mathrm{loc}} u C^{\alpha}(G)_{\mathrm{loc}}$ совпадают.

Замечание 2. В доказательстве теорем 1 и 2 существенно используются теорема Де Джорджи и Нэша и теорема Литтмана, Стампаккьи и Вайнбергера о существовании функции Грина для оператора $L$ в шаре, которая на его 
компактных подмножествах слабо эквивалентна функции Грина для оператора Лапласа $\Delta$. Первая из этих теорем справедлива без условия симметричности матрицы коэффициентов оператора $L$ почти всюду в области $G$, а во второй это условие можно опустить по крайней мере при $n \geqslant 3$ (см. [3]). Поэтому теоремы 1 и 2 и все приводимые ниже рассуждения будут справедливыми при $n \geqslant 3$ без требования симметричности матрицы коэффициентов оператора $L$ почти всюду в области $G$.

Остановимся на истории вопроса. Описанию устранимых особенностей для решений линейных дифференциальных уравнений с гладкими коэффициентами в классах Гёльдера и их различных обобщениях посвящено большое количество работ (см., например, [4]-[16]). В частности, для многих эллиптических уравнений устранимые особенности в таких классах полностью описываются в терминах мер Хаусдорфа. Подобные результаты имеют место и для некоторых квазилинейных эллиптических уравнений (см. [17]-[19]). Используемый в настоящей работе подход к описанию функциональных классов восходит к работе Федорова [20], в которой фактически рассматриваются классы функций, непрерывных в области $G \subset \mathbb{C}$, определяемые с помощью локальных аппроксимаций в равномерной метрике голоморфными функциями. В дальнейшем идея такого рода определения функциональных классов использовалась в работах Ищанова и автора (см. [11], [16], [18], [21]). Отметим (см. [22]), что для оператора Лапласа $\Delta$ при всех $\alpha \in(0,1)$ имеют место равенства $U_{\Delta}^{\alpha}(G)_{\text {loc }}=C_{\Delta}^{\alpha}(G)_{\text {loc }}=C^{\alpha}(G)_{\text {loc }}$, $U_{\Delta}^{1+\alpha}(G)_{\mathrm{loc}}=C_{\Delta}^{1+\alpha}(G)_{\mathrm{loc}}=C^{1, \alpha}(G)_{\mathrm{loc}}, U_{\Delta}^{1}(G)_{\mathrm{loc}}=C_{\Delta}^{1}(G)_{\mathrm{loc}}=Z(G)_{\mathrm{loc}}$. Поэтому из теоремы 1 вытекают известные результаты об устранимых особенностях гармонических функций в классах Гёльдера-Зигмунда, установленные в работах [5]-[7], [12], [13].

Работа организована следующим образом. В $\S 1$ напоминаются необходимые определения и устанавливается ряд вспомогательных результатов. Второй и третий параграфы работы посвящены доказательству соответственно теорем 1 и 2 .

\section{§1. Определения и вспомогательные результаты}

Напомним, что (внешней) хаусдорфовой мерой $\operatorname{mes}^{\alpha} E$ порядка $\alpha(\alpha \geqslant 0)$ множества $E \subset \mathbb{R}^{n}$ называется конечный или равный $+\infty$ предел при $t \rightarrow 0$ величины $\inf \left(\sum_{i} r_{i}^{\alpha}\right)$, где инфимум берется по всем не более чем счетным системам открытых шаров $\left\{B\left(x_{i}, r_{i}\right)\right\}_{i}$ с $r_{i} \leqslant t$, образующих покрытие множества $E$.

Под обобщенным решением уравнения $L f=0$ в области $G$ мы понимаем, как обычно, функцию $u \in W_{2}^{1}(G)_{\text {loc }}$, такую, что для каждой бесконечно дифференцируемой функции $\varphi$ с компактным носителем в $G\left(\varphi \in C_{0}^{\infty}(G)\right)$ выполняется равенство

$$
\sum_{i, j=1}^{n} \int_{G} a_{i j}(x) D_{i} u(x) D_{j} \varphi(x) d x=0 .
$$

Пусть $f \in C(G)$ и $r>0$ выбрано так, что множество $G_{r}$ непусто. Тогда имеет место следующая

Лемма 1. Функиия $f_{L, r}$ непрерьвна на множестве $G_{r}$.

Доказательство. Фиксируем шар $B(x, r) \Subset G$. Тогда существует такое $\delta_{0} \in$ $(0, r / 2)$, что $B\left(x, r+\delta_{0}\right) \Subset G$. Пусть $\omega(\delta), 0 \leqslant \delta \leqslant \delta_{0}$, обозначает модуль 
непрерывности функции $f$ на шаре $B\left(x, r+\delta_{0}\right)$. Из теоремы В. Г. Мазьи об оценке модуля непрерывности решения задачи Дирихле для уравнения $L u=0$ в регулярной граничной точке (см. [23, теорема 1.34]) следует существование непрерывной и неубывающей на отрезке $\left[0, \delta_{0}\right]$ функции $g(\delta)$, которая обладает следующими свойствами: $g(0)=0$; для всех $d \in\left[r, r+\delta_{0}\right], t_{1} \in S(x, d)$ и $t_{2} \in$ $B(x, d)$, таких, что $\left|t_{1}-t_{2}\right| \leqslant \delta_{0}$, выполняется неравенство $\left|f\left(t_{1}\right)-f_{L, x, d}\left(t_{2}\right)\right| \leqslant$ $\omega\left(\left|t_{1}-t_{2}\right|\right)+g\left(\left|t_{1}-t_{2}\right|\right)$.

Зафиксируем произвольным образом $\varepsilon>0$ и выберем такое $\delta \in\left(0, \delta_{0} / 2\right)$, чтобы выполнялось неравенство $2 \omega(2 \delta)+g(2 \delta) \leqslant \varepsilon / 4$. Возьмем $\eta_{0}>0$ таким, чтобы любая сфера $S(y, r)$ с центром $y \in B\left(x, \eta_{0}\right)$ попадала в замкнутое кольцо $\bar{B}(x, r+\delta) \backslash B(x, r-\delta)$. Поскольку любая точка этого кольца удалена от сферы $S(x, r+\delta)$ на расстояние, не превосходящее $2 \delta$, то на нем максимум модуля функции $f-f_{L, x, r+\delta}$ не превосходит $\omega(2 \delta)+(\omega(2 \delta)+g(2 \delta)) \leqslant \varepsilon / 4$. По принципу максимума для решений рассматриваемого уравнения отсюда заключаем, что для всех $y \in B\left(x, \eta_{0}\right)$ выполняется неравенство $\left|f_{L, r}(y)-f_{L, x, r+\delta}(y)\right| \leqslant \varepsilon / 4$; следовательно, выбирая $\eta \in\left(0, \eta_{0}\right]$ таким, что $\operatorname{osc}_{B(x, \eta)} f_{L, x, r+\delta} \leqslant \varepsilon / 2$, мы при каждом $y \in B(x, \eta)$ имеем цепочку неравенств

$$
\begin{aligned}
\left|f_{L, r}(y)-f_{L, r}(x)\right| \leqslant\left|f_{L, x, r+\delta}(x)-f_{L, r}(x)\right|+\left|f_{L, x, r+\delta}(y)-f_{L, x, r+\delta}(x)\right| & \\
& +\left|f_{L, x, r+\delta}(y)-f_{L, r}(y)\right| \leqslant \frac{\varepsilon}{4}+\underset{B(x, \eta)}{\operatorname{Osc}} f_{L, x, r+\delta}+\frac{\varepsilon}{4} \leqslant \varepsilon,
\end{aligned}
$$

которая и означает непрерывность функции $f_{L, r}$ в точке $x$.

Лемма 1 доказана.

Напомним теперь понятия $L$-гармонической меры и функиии Грина для оператора $L$, играющие ключевую роль в последующих рассуждениях. Мы приводим соответствующие формулировки не в максимальной общности, а для тех случаев, которые нам потребуются в дальнейшем.

Если $B(x, r) \Subset G$, то отображение, каждой функции $f \in C(S(x, r))$ ставящее в соответствие число $f_{L, x, r}(x)$, является непрерывным линейным функционалом на $C(S(x, r))$, который принимает неотрицательные значения на неотрицательных функциях $f$. Поэтому имеет место представление

$$
f_{L, x, r}(x)=\int_{S(x, r)} f(y) d \omega_{L, x, r}(y),
$$

где $\omega_{L, x, r}$ - вероятностная борелевская мера на сфере $S(x, r)$. Мы будем называть эту меру $L$-гармонической мерой на сфере $S(x, r)$. Если $L-$ оператор Лапласа, то $L$-гармоническая мера называется, как обычно, гармонической и с точностью до постоянного множителя совпадает с $(n-1)$-мерной мерой Лебега на сфере $S(x, r)$. В то же время в случае произвольного оператора $L$ $(n-1)$-мерная мера Лебега и $L$-гармоническая мера на сфере $S(x, r)$ не являются абсолютно непрерывными по отношению друг к другу: соответствующие примеры, в которых $L$ - оператор с непрерывными коэффициентами, приведены в работе [24].

В дальнейшем будем считать, что оператор $L$ задан на всем пространстве $\mathbb{R}^{n}$, доопределяя, если нужно, его коэффициенты в точках $x \in \mathbb{R}^{n} \backslash G$ равенствами $a_{i i}(x)=\lambda, a_{i j}(x)=0, i, j=1, \ldots, n, i \neq j$.

Функцией Грина для оператора $L$ в шаре $B\left(x_{0}, r\right)$ называется функция $G_{L, x_{0}, r}(x, y)$, определенная на множестве $\left\{x, y \in B\left(x_{0}, r\right): x \neq y\right\}$, которая 
непрерывна и положительна на нем и при каждом фиксированном $y_{0} \in B\left(x_{0}, r\right)$ удовлетворяет (как функция от $x$ ) следующим условиям: (a) $G_{L, x_{0}, r}\left(x, y_{0}\right) \in$ $W_{1}^{1}\left(B\left(x_{0}, r\right)\right)$, причем в каждой подобласти, полученной из $B\left(x_{0}, r\right)$ выбрасыванием шара $\bar{B}\left(y_{0}, \varepsilon\right) \subset B\left(x_{0}, r\right)(\varepsilon>0)$, все первые обобщенные производные функции $G_{L, x_{0}, r}\left(x, y_{0}\right)$ суммируемы в квадрате; (b) в каждой граничной точке шара $B\left(x_{0}, r\right)$ функция $G_{L, x_{0}, r}\left(x, y_{0}\right)$ имеет предельные значения, равные нулю (ниже мы считаем, что функция $G_{L, x_{0}, r}\left(x, y_{0}\right)$ доопределена нулем при $\left.x \in S\left(x_{0}, r\right)\right) ;\left(\right.$ с) для любой функции $\varphi \in C_{0}^{\infty}\left(B\left(x_{0}, r\right)\right)$ справедливо равенство

$$
\sum_{i, j=1}^{n} \int_{B\left(x_{0}, r\right)} a_{i j}(x) D_{i} G_{L, x_{0}, r}\left(x, y_{0}\right) D_{j} \varphi(x) d x=\varphi\left(y_{0}\right) .
$$

Известная теорема Литтмана, Стампаккьи и Вайнбергера [25] утверждает, что такая функция $G_{L, x_{0}, r}$ существует и единственна при любых $x_{0} \in \mathbb{R}$ и $r>0$ и на компактных подмножествах шара $B\left(x_{0}, r\right)$ она слабо эквивалентна функции Грина для оператора Лапласа $\Delta$ : для каждого $t \in(0,1)$ существуют такие $C_{1}, C_{2}>0$, зависящие только от $t$, размерности $n$ и постоянных эллиптичности $\lambda$ и $\Lambda$ оператора $L$, что для всех $x, y \in B\left(x_{0}, t r\right), x \neq y$, справедливо двойное неравенство

$$
C_{1} G_{\Delta, x_{0}, r}(x, y) \leqslant G_{L, x_{0}, r}(x, y) \leqslant C_{2} G_{\Delta, x_{0}, r}(x, y) .
$$

Отсюда, в частности, следует, что при $x=y \in B\left(x_{0}, r\right)$ мы можем доопределить функцию $G_{L, x_{0}, r}$ по непрерывности равенством $G_{L, x_{0}, r}(x, y)=+\infty$, и в дальнейшем такое доопределение всегда предполагается. При фиксированном $y_{0} \in B\left(x_{0}, r\right)$ мы, как обычно, называем функцию $G_{L, x_{0}, r}\left(x, y_{0}\right)$ функцией Грина для оператора $L$ в шаре $B\left(x_{0}, r\right)$ с полюсом в точке $y_{0}$.

Пусть $O$ обозначает начало координат в $\mathbb{R}^{n}$. Из определения функции Грина следует, что для всех $x_{0} \in \mathbb{R}^{n}$ и $r>0$ функция $G_{L, x_{0}, r}\left(x_{0}+x, x_{0}+y\right), x, y \in$ $B(O, r)$, совпадает с функцией $G_{L_{0}, O, r}(x, y)$, а функция $r^{n-2} G_{L, O, r}(r x, r y), x, y \in$ $B(O, 1)$, совпадает с функцией $G_{L_{1}, O, 1}(x, y)$, где операторы $L_{0}$ и $L_{1}$ заданы соответственно матрицами коэффициентов $\left\|a_{i j}\left(x_{0}+x\right)\right\|$ и $\left\|a_{i j}(r x)\right\|\left(x \in \mathbb{R}^{n}\right.$, $i, j=1, \ldots, n)$. Поскольку эти операторы имеют те же самые константы эллиптичности, что и $L$, то из приведенной выше теоремы Литтмана, Стампаккьи и Вайнбергера вытекает, что для всех $x_{0} \in \mathbb{R}^{n}$ и $r>0$ имеет место неравенство

$$
G_{L, x_{0}, r}(x, y) \geqslant C r^{2-n}, \quad x, y \in B\left(x_{0}, r / 2\right),
$$

где $C>0$ зависит только от $n, \lambda$ и $\Lambda$.

Зафиксируем $R>0$ так, что $G \Subset B(O, R)$, и положим $g(x, y):=G_{L, O, 4 R}(x, y)$, $x, y \in B(O, 4 R)$. В следующей лемме собраны некоторые нужные нам свойства $L$-гармонической емкости и функции Грина.

Лемма 2. Каковъ бъ ни бъли точка $y_{0} \in B(O, R)$ и иар $B\left(x_{0}, r\right) \Subset B(O, R)$ $(r>0)$, справедливы следующие утвержсдения:

(а) функиия $g\left(x, y_{0}\right)$ суммируема на сфере $S\left(x_{0}, r\right)$ по мере $\omega_{L, x_{0}, r}$, а интеграл $\int_{S\left(x_{0}, r\right)} g\left(x, y_{0}\right) d \omega_{L, x_{0}, r}(x)$ ограничен сверху положстельной величиной, не зависящей от точек $x_{0}$ и $y_{0}$;

(b) имеет место неравенство

$$
g\left(x_{0}, y_{0}\right)-\int_{S\left(x_{0}, r\right)} g\left(x, y_{0}\right) d \omega_{L, x_{0}, r}(x) \geqslant 0
$$


(с) существует такое $C>0$, зависящее только от оператора L, что при $y_{0} \in B\left(x_{0}, r / 2\right) \backslash\left\{x_{0}\right\}$ выполняется неравенство

$$
g\left(x_{0}, y_{0}\right)-\int_{S\left(x_{0}, r\right)} g\left(x, y_{0}\right) d \omega_{L, x_{0}, r}(x) \geqslant C r^{2-n} .
$$

Доказательство. Поскольку в области $B(O, 4 R) \backslash\left\{y_{0}\right\}$ функция $g\left(x, y_{0}\right)$ является обобщенным решением уравнения $L f=0$, она в этой области непрерывна и, следовательно, суммируема по $L$-гармонической мере на любой сфере, лежащей в этой области и не содержащей точку $y_{0}$. Рассмотрим случай $y_{0} \in S\left(x_{0}, r\right)$. Возьмем сходящуюся к точке $y_{0}$ последовательность $\left\{y_{k}\right\}_{k=1}^{\infty} \subset$ $B(O, R) \backslash \bar{B}\left(x_{0}, r\right)$. Тогда функции $g\left(x, y_{k}\right), k \in \mathbb{N}:=\{1,2, \ldots\}$, суммируемы по мере $\omega_{L, x_{0}, r}$ и, поскольку они принадлежат классу $A_{L}\left(B\left(x_{0}, r\right)\right)$, справедливы равенства

$$
\int_{S\left(x_{0}, r\right)} g\left(x, y_{k}\right) d \omega_{L, x_{0}, r}(x)=g\left(x_{0}, y_{k}\right), \quad k \in \mathbb{N} .
$$

Так как последовательность $\left\{g\left(x_{0}, y_{k}\right)\right\}_{k=1}^{\infty}$ сходится к $g\left(x_{0}, y_{0}\right)$, то суммируемость функции $g\left(x, y_{0}\right)$ по мере $\omega_{L, x_{0}, r}$ и равенство

$$
\int_{S\left(x_{0}, r\right)} g\left(x, y_{0}\right) d \omega_{L, x_{0}, r}(x)=g\left(x_{0}, y_{0}\right)
$$

вытекают из теоремы Фату о предельном переходе под знаком интеграла Лебега.

Если $y_{0} \notin B\left(x_{0}, r\right)$, то справедливо равенство (2) и неравенство

$$
\int_{S\left(x_{0}, r\right)} g\left(x, y_{0}\right) d \omega_{L, x_{0}, r}(x) \leqslant \sup _{x, y \in B(O, R):|x-y| \geqslant r} g(x, y),
$$

которое, очевидно, выполнено и в случае $x_{0}=y_{0}$. Если же $y_{0} \in B\left(x_{0}, r\right) \backslash\left\{x_{0}\right\}$, то существует такое $C \geqslant 1$, зависящее только от $n, \lambda$ и $\Lambda$, что при всех $x \in S\left(x_{0}, r\right)$ имеет место неравенство

$$
g\left(x, y_{0}\right) \leqslant C\left(g\left(x, z_{0}\right)+g\left(x, x_{0}\right)\right),
$$

где $z_{0}$ - точка пересечения прямой, проходящей через $x_{0}$ и $y_{0}$, со сферой $S\left(x_{0}, r\right)$. Для случая $L=\Delta$ это следует из явных формул для функции Грина в шаре, общий случай сводится к случаю $L=\Delta$ с помощью теоремы Литтмана, Стампаккьи и Вайнбергера. Поэтому из (3) и (4) получаем неравенство

$$
\int_{S\left(x_{0}, r\right)} g\left(x, y_{0}\right) d \omega_{L, x_{0}, r}(x) \leqslant 2 C \sup _{x, y \in B(O, R):|x-y| \geqslant r} g(x, y)
$$

$\left(B\left(x_{0}, r\right) \Subset B(O, R), y_{0} \in B(O, R)\right)$. Поскольку его правая часть конечна и не зависит от $x_{0}$ и $y_{0}$, то мы доказали утверждение (a).

При $y_{0} \notin B\left(x_{0}, r\right)$ неравенство в утверждении (b) превращается в равенство. Если же $y_{0} \in B\left(x_{0}, r\right)$, то правая часть этого неравенства совпадает с функцией $G_{L, x_{0}, r}\left(x, y_{0}\right)$ и утверждение $(\mathrm{b})$ следует из положительности функции Грина, а (с) 一 из (1).

Лемма 2 доказана. 
Доказательство следующей леммы основано на рассуждениях, предложенных в [13]. Она играет ключевую роль при доказательстве достаточности в теореме 1 .

Лемма 3. Пусть $0<\alpha \leqslant 2 u$ mes $^{n-2+\alpha} K=0$. Тогда в шаре $B(O, R)$ существует полунепрерывная снизу положительная функиия $h \in L(B(O, R))$, суммируемая по мере $\omega_{L, x_{0}, r}$ на каждой сфере $S\left(x_{0}, r\right) \subset B(O, R)$, являющаяся обобщенным решением уравнения $L f=0$ на множестве $B(O, R) \backslash K$ и такая, что для любой точки $x \in K$ имеет место равенство

$$
\varlimsup_{r \rightarrow 0} r^{-\alpha}\left(h(x)-\int_{S(x, r)} h(y) d \omega_{L, x, r}(y)\right)=+\infty .
$$

Доказательство. Пусть выполнены условия леммы. Для каждого $m \in \mathbb{N}$ выберем конечное число шаров $B\left(x_{m, 1}, r_{m, 1}\right), \ldots, B\left(x_{m, k_{m}}, r_{m, k_{m}}\right)$, таких, что $K \subset \bigcup_{k=1}^{k_{m}} B\left(x_{m, k}, r_{m, k}\right) \subset \bigcup_{k=1}^{k_{m}} B\left(x_{m, k}, 2 r_{m, k}\right) \Subset G$ и $\sum_{k=1}^{k_{m}}\left(r_{m, k}\right)^{n-2+\alpha} \leqslant 4^{-m}$. Определим функцию $h(x), x \in B(O, R)$, равенством

$$
h(x):=\sum_{m=1}^{\infty}\left(2^{m} \sum_{k=1}^{k_{m}}\left(r_{m, k}\right)^{n-2+\alpha} g\left(x, x_{m, k}\right)\right),
$$

Так как ряд $\sum_{m=1}^{\infty}\left(2^{m} \sum_{k=1}^{k_{m}}\left(r_{m, k}\right)^{n-2+\alpha}\right)$ сходится, $\sup _{y \in B(O, R)} \int_{B(O, R)} g(x, y) d x$ - конечная величина, а каждая из функций $g\left(x, x_{m, k}\right)$ полунепрерывна снизу в $B(O, R)$, то функция $h(x)$ полунепрерывна снизу в $B(O, R)$ как предел возрастающей последовательности полунепрерывных снизу функций и по теореме Б. Леви о предельном переходе под знаком интеграла Лебега суммируема по мере Лебега в $B(O, R)$. Из теоремы Леви и утверждения (а) леммы 2 также следует интегрируемость функции $h$ по мере $\omega_{L, x_{0}, r}$ на каждой сфере $S\left(x_{0}, r\right) \subset B(O, R)$. Применяя теорему Литтмана, Стампаккьи и Вайнбергера, заключаем, что ряд в правой части формулы (6) сходится равномерно на компактных подмножествах открытого множества $B(O, R) \backslash K$ и, следовательно, его сумма $h(x)$ является обобщенным решением уравнения $L f=0$ в $B(O, R) \backslash K$.

Докажем последнее утверждение леммы. Пусть $x \in K$. Для каждого натурального $m$ выберем $k \in\left\{1, \ldots, k_{m}\right\}$ так, чтобы выполнялось условие $x \in$ $B\left(x_{m, k}, r_{m, k}\right)$. Тогда, пользуясь утверждениями (b) и (c) леммы 2 , имеем соотношения

$$
\begin{aligned}
& \left(2 r_{m, k}\right)^{-\alpha}\left(h(x)-\int_{S(x, 2 r)} h(y) d \omega_{L, x, r}(y)\right) \\
& \geqslant\left(2 r_{m, k}\right)^{-\alpha} 2^{m}\left(r_{m, k}\right)^{n-2+\alpha}\left(g\left(x, x_{m, k}\right)-\int_{S(x, 2 r)} g\left(y, x_{m, k}\right) d \omega_{L, x, r}(y)\right) \\
& \quad \geqslant C\left(2 r_{m, k}\right)^{-\alpha} 2^{m}\left(r_{m, k}\right)^{n-2+\alpha}\left(2 r_{m, k}\right)^{2-n}=C 2^{m-(n-2+\alpha)}
\end{aligned}
$$

с тем же самым $C>0$, что и в утверждении (с) леммы 2 . При $m \rightarrow \infty$ отсюда получаем (5).

Лемма доказана.

В доказательстве теоремы 2 будет использована следующая

Лемма 4 (см. [26, с. 86]). Пусть ф(t) - неотрицательная и неубывающая функиия аргумента $t>0,0<\alpha<\gamma$, и пусть $R_{0}>0$. Кроме того, пусть при 
всех $r, R>0, r<R \leqslant R_{0}$, выполнено неравенство $\phi(r) \leqslant A R^{\alpha}+B(r / R)^{\gamma} \phi(R)$, где $A, B>0$. Тогда существует такое $C>0$, зависящее только от $B, \alpha$ u $\gamma$, что при всех $r, R \in\left(0, R_{0}\right], r<R$, имеет место неравенство $\phi(r) \leqslant$ $C\left[A r^{\alpha}+(r / R)^{\alpha} \phi(R)\right]$.

\section{§2. Доказательство теоремы 1}

Достаточность доказывается с помощью рассуждений из работы [13].

Пусть $0<\alpha \leqslant 2$, mes ${ }^{n-2+\alpha} K=0$, и пусть $u$ - функция из класса $U_{L}^{\alpha}(G)$, которая является обобщенным решением уравнения $L f=0$ на множестве $G \backslash K$. По лемме 3 существует функция $h \in L(B(O, R))$, удовлетворяющая всем условиям, перечисленным в формулировке этой леммы. Выберем область $G_{1}$ так, что $K \subset G_{1} \Subset G$ и ее граница $\partial G_{1}$ регулярна для уравнения Лапласа. Тогда в замкнутой области $\bar{G}_{1}$ существует единственная непрерывная функция $w(x)$, определяемая условиями $w \in A_{L}\left(G_{1}\right), w(x) \equiv u(x)$ на $\partial G_{1}$. Рассмотрим семейство функций $u_{\varepsilon}(x):=u(x)-w(x)+\varepsilon h(x), x \in \bar{G}_{1}$, зависящее от вещественного параметра $\varepsilon$. Пусть $\varepsilon>0$. Тогда функция $u_{\varepsilon}$ полунепрерывна снизу на $\bar{G}_{1}$, непрерывна на $\bar{G}_{1} \backslash K$, является обобщенным решением уравнения $L f=0$ в $G_{1} \backslash K$, суммируема по мере $\omega_{L, x_{0}, r}$ на каждой сфере $S\left(x_{0}, r\right) \subset G_{1}$ и для любой точки $x \in K$ имеет место равенство

$$
\varlimsup_{r \rightarrow 0} r^{-\alpha}\left(u_{\varepsilon}(x)-\int_{S(x, r)} u_{\varepsilon}(y) d \omega_{L, x, r}(y)\right)=+\infty .
$$

Отсюда вытекает, что функция $u_{\varepsilon}$ достигает своего минимума на $\bar{G}_{1}$ в какой-то точке границы области $G_{1}$. С другой стороны, $u_{\varepsilon}(x)>0$ для всех $x \in \partial G_{1}$ и, следовательно, функция $u_{\varepsilon}$ положительна в области $G_{1}$. Предельным переходом при $\varepsilon \rightarrow+0$ отсюда получаем, что $u_{0}(x) \geqslant 0$ для любого $x \in G_{1}$.

Точно так же устанавливается, что при $\varepsilon<0$ функция $u_{\varepsilon}$ принимает только отрицательные значения в $G_{1}$, откуда предельным переходом при $\varepsilon \rightarrow-0$ получаем, что $u_{0}(x) \leqslant 0$ для любого $x \in G_{1}$.

Таким образом, функция $u_{0}$ одновременно неотрицательна и неположительна в $G_{1}$. Следовательно, она тождественно равна там нулю и, значит, $u(x)=$ $w(x)$ для любого $x \in G_{1}$. Так как $w \in A_{L}\left(G_{1}\right)$, то $u \in A_{L}(G)$.

Достаточность условия mes $^{n-2+\alpha} K=0$ в теореме 1 доказана. Установим его необходимость.

Пусть mes $^{n-2+\alpha} K>0$. В случае $\operatorname{mes}^{n} K>0$ можно без уменьшения общности считать, что множество $K$ не содержит внутренних точек (выбрасывая в случае необходимости из $K$ счетное число попарно не пересекающихся открытых шаров так, что сумма их хаусдорфовых мер порядка $n$ меньше, чем $\operatorname{mes}^{n} K$, и каждая внутренняя точка множества $K$ является предельной для объединения удаляемых шаров). По лемме Фростмана (см. [8]) существует борелевская мера $\mu$ с носителем на $K$, такая, что $\mu(K)>0$ и

$$
\mu(B(x, r)) \leqslant r^{n-2+\alpha}
$$

для всех $x \in \mathbb{R}^{n}$ и $r>0$. Определим функцию

$$
u(x):=\int_{K} g(x, y) d \mu(y), \quad x \in B(O, 4 R),
$$


и покажем, что $u \in U_{L}^{\alpha}(G)$. Фиксируем произвольный шар $B\left(x_{0}, 4 r\right) \Subset B(O, R)$ с $r \in(0,1]$. Пусть $u_{1}(x):=\int_{K \backslash B\left(x_{0}, 2 r\right)} g(x, y) d \mu(y)$ для непустого множества $K \backslash B\left(x_{0}, 2 r\right), u_{1}(x):=0$ при $K \backslash B\left(x_{0}, 2 r\right)=\varnothing$ и $u_{2}(x):=\int_{B\left(x_{0}, 2 r\right)} g(x, y) d \mu(y)$ $(x \in B(O, 4 R))$. Тогда $u_{1} \in A_{L}\left(B\left(x_{0}, r\right)\right)$ и

$$
u(x)=u_{1}(x)+u_{2}(x) \quad \forall x \in B(O, 4 R) .
$$

Пусть $x \in B\left(x_{0}, r\right)$. Используя (7), явные формулы для функции Грина для оператора Лапласа в шаре с полюсом в его центре, оценку сверху функции Грина для оператора $L$ из теоремы Литтмана, Стампаккьи и Вайнбергера, включения $B\left(x_{0}, 2 r\right) \subset B(x, 3 r)$ и $B(O, 4 R) \subset B(x, 5 R)$ и вытекающее из принципа максимума увеличение значений функции Грина с расширением области, получаем

$$
\begin{aligned}
\left|u_{2}(x)\right| & =\int_{B\left(x_{0}, 2 r\right)} g(x, y) d \mu(y) \leqslant \int_{B(x, 3 r)} g(x, y) d \mu(y) \\
& \leqslant \sum_{k=0}^{\infty} \int_{B\left(x, 3 r / 2^{k}\right) \backslash B\left(x, 3 r / 2^{k+1}\right)} g(x, y) d \mu(y) \\
& \leqslant C_{1} \sum_{k=0}^{\infty}\left(\frac{3 r}{2^{k+1}}\right)^{2-n} \mu\left(B\left(x, \frac{3 r}{2^{k}}\right)\right) \\
& \leqslant C_{1} \sum_{k=0}^{\infty}\left(\frac{3 r}{2^{k+1}}\right)^{2-n}\left(\frac{3 r}{2^{k}}\right)^{n-2+\alpha}=C_{2} r^{\alpha}, \quad n \geqslant 3, \\
\left|u_{2}(x)\right| & \leqslant \sum_{k=0}^{\infty} \int_{B\left(x, 3 r / 2^{k}\right) \backslash B\left(x, 3 r / 2^{k+1}\right)} g(x, y) d \mu(y) \\
& \leqslant C_{1} \sum_{k=0}^{\infty} \log \frac{5 R 2^{k+1}}{3 r}\left(\frac{3 r}{2^{k}}\right)^{\alpha} \quad n=2 . \\
& \leqslant C_{1}\left(\sup _{t \geqslant 1} \frac{\log t}{t^{\alpha / 2}}\right)_{k=0}^{\infty}\left(\frac{5 R 2^{k+1}}{3 r}\right)^{\alpha / 2}\left(\frac{3 r}{2^{k}}\right)^{\alpha}=C_{2} r^{\alpha / 2}, \quad n=2 .
\end{aligned}
$$

где положительные величины $C_{1}$ и $C_{2}$ зависят только от $n, \lambda, \Lambda$ и $\alpha$, а при $n=2$ - и от $R$. Из этих оценок и из (8) вытекает, что функция $u$ ограничена сверху в некоторой окрестности компакта $K$ и, поскольку она непрерывна в $B(O, 4 R) \backslash K$, величина $\sup _{B(O, R)} u$ конечна. Из приведенных оценок и из (8) также получаем

$$
\sup _{B\left(x_{0}, r\right)}\left|u-u_{1}\right|=\sup _{B\left(x_{0}, r\right)}\left|u_{2}\right| \leqslant C_{2} r^{\alpha / 2}
$$

и при $n \geqslant 3$

$$
\sup _{B\left(x_{0}, r\right)}\left|u-u_{1}\right| \leqslant C_{2} r^{\alpha} .
$$

Поскольку $u_{1} \in A_{L}\left(B\left(x_{0}, 2 r\right)\right)$, по теореме Де Джорджи и Нэша имеем неравенство $\operatorname{osc}_{B\left(x_{0}, r^{2}\right)} u_{1} \leqslant C\left(r^{2} / r\right)^{\gamma} \operatorname{osc}_{B\left(x_{0}, r\right)} u_{1}$, где $C$ не зависит от $r$. Отсюда для 
всех $x \in B\left(x_{0}, r^{2}\right)$ получаем

$$
\begin{aligned}
\left|u(x)-u\left(x_{0}\right)\right| & \leqslant\left|u(x)-u_{1}(x)\right|+\left|u_{1}(x)-u_{1}\left(x_{0}\right)\right|+\left|u_{1}\left(x_{0}\right)-u\left(x_{0}\right)\right| \\
& \leqslant 2 C_{2} r^{\alpha / 2}+\underset{B\left(x_{0}, r^{2}\right)}{\operatorname{Osc}} u_{1} \leqslant 2 C_{2} r^{\alpha / 2}+C r^{\gamma} \underset{B\left(x_{0}, r\right)}{\text { osc }} u_{1} \\
& \leqslant 2 C_{2} r^{\alpha / 2}+C r^{\gamma}\left(2 \sup _{B\left(x_{0}, r\right)}\left|u-u_{1}\right|+2 \sup _{B(O, R)} u\right) \\
& \leqslant 2 C_{2} r^{\alpha / 2}+C r^{\gamma}\left(2 C_{2} r^{\alpha / 2}+2 \sup _{B(O, R)} u\right) .
\end{aligned}
$$

Следовательно, $\left|u(x)-u\left(x_{0}\right)\right| \rightarrow 0$ при $x \rightarrow x_{0}$. Поскольку точка $x_{0} \in B(O, R)$ в приведенных рассуждениях выбиралась произвольным образом, тем самым установлена непрерывность потенциала $u$ в шаре $B(O, R)$.

Рассмотрим более подробно случай $n=2$. Пусть

$$
u_{3}(x):=\int_{B\left(x_{0}, 2 r\right)} G_{L, x_{0}, 4 r}(x, y) d \mu(y), \quad x \in B\left(x_{0}, 2 r\right) .
$$

Тогда $u_{2}-u_{3} \in A_{L}\left(B\left(x_{0}, 2 r\right)\right)$ и, повторяя проведенные выше рассуждения, основанные на применении теоремы Литтмана, Стампаккьи и Вайнбергера, для каждого $x \in B\left(x_{0}, 2 r\right)$ имеем

$$
\begin{aligned}
u_{2}(x) & -\left(u_{2}(x)-u_{3}(x)\right)=u_{3}(x)=\int_{B\left(x_{0}, 2 r\right)} G_{L, x_{0}, 4 r}(x, y) d \mu(y) \\
& \leqslant C_{1} \int_{B\left(x_{0}, 2 r\right)} G_{\Delta, x_{0}, 4 r}(x, y) d \mu(y) \leqslant C_{1} \int_{B\left(x_{0}, 2 r\right)} G_{\Delta, x, 6 r}(x, y) d \mu(y) \\
& \leqslant C_{1} \int_{B(x, 4 r)} \log \frac{6 r}{|x-y|} d \mu(y)=C_{1} \sum_{k=0}^{\infty} \int_{B\left(x, 4 r / 2^{k}\right) \backslash B\left(x, 4 r / 2^{k+1}\right)} \log \frac{6 r}{|x-y|} d \mu(y) \\
& \leqslant C_{1} \sum_{k=0}^{\infty} \log \frac{6 r}{4 r / 2^{k+1}}\left(4 r / 2^{k}\right)^{\alpha} \leqslant C_{1} r^{\alpha} \sum_{k=0}^{\infty}\left(\log 2^{k+2}\right)\left(2^{2-k}\right)^{\alpha}=C_{2} r^{\alpha},
\end{aligned}
$$

где положительные величины $C_{1}$ и $C_{2}$ зависят только от $n, \lambda, \Lambda$ и $\alpha$. Из этих оценок получаем неравенство

$$
\left\|u_{2}-\left(u_{2}-u_{3}\right) \mid C\left(B\left(x_{0}, r\right)\right)\right\| \leqslant C_{2} r^{\alpha} .
$$

Поскольку $G \Subset B(O, R)$, найдется такое $r_{0} \in(0,1]$, что для всех $x \in G$ имеет место включение $B\left(x, 4 r_{0}\right) \Subset B(O, R)$. Отсюда, учитывая $(9)$ и $(10)$, для всех $r \in\left(0, r_{0}\right]$ имеем неравенство

$$
\left\|u-w \mid C\left(B\left(x_{0}, r\right)\right)\right\| \leqslant C_{2} r^{\alpha}
$$

где $w(x):=u_{1}(x)$ при $n \geqslant 3$ и $w(x):=u_{1}(x)+\left(u_{2}(x)-u_{3}(x)\right)$ при $n=2$, а $C_{2}$ положительно и зависит только от $n, \lambda, \Lambda$ и $\alpha$. Поскольку $w \in A_{L}\left(B\left(x_{0}, 2 r\right)\right)$, применяя принцип максимума, получаем из (11) неравенство

$$
\left\|u_{L, x_{0}, r}-w \mid C\left(B\left(x_{0}, r\right)\right)\right\| \leqslant C_{2} r^{\alpha} .
$$

Из (11) и (12), учитывая произвол при выборе $x_{0}$ в наших рассуждениях, заключаем, что для каждого шара $B\left(x_{0}, r\right) \subset G$ с $0<r \leqslant r_{0}$ имеет место неравенство

$$
\left\|u-u_{L, x_{0}, r} \mid C\left(B\left(x_{0}, r\right)\right)\right\| \leqslant 2 C_{2} r^{\alpha},
$$


которое и означает, что $u \in U_{L}^{\alpha}(G)$. Поскольку множество $K$ не имеет внутренних точек, функция $u$ не может быть продолжена с $G \backslash K$ на $G$ как обобщенное решение уравнения $L f=0$ : если бы такое продолжение существовало, то по непрерывности при всех $x \in K$ оно совпадало бы с $u(x)$, но $u \notin A_{L}(G)(\mathrm{cm}$. $[25])$.

Теорема доказана.

\section{§3. Доказательство теоремы 2}

Как уже отмечалось, включение $C^{\alpha}(G) \subset U_{L}^{\alpha}(G)$ при $0<\alpha<1$ следует из принципа максимума для решений уравнения $L f=0$. Пусть $\gamma-$ гёльдеров показатель в приведенной выше формулировке теоремы Де Джорджи и Нэша, и пусть $u \in U_{L}^{\alpha}(G)$. Тогда для любой пары концентрических шаров $B(x, r) \Subset$ $B(x, R) \Subset G$ имеют место неравенства

$$
\begin{aligned}
\underset{B(x, r)}{\operatorname{Osc}} u & \leqslant 2 \sup _{B(x, r)}\left|u-u_{L, x, R}\right|+\underset{B(x, r)}{\operatorname{osc}} u_{L, x, R} \\
& \leqslant 2 A R^{\alpha}+\underset{B(x, r)}{\operatorname{osc}} u_{L, x, R} \leqslant 2 A R^{\alpha}+C\left(\frac{r}{R}\right)^{\gamma} \underset{B(x, R)}{\operatorname{Osc}} u_{L, x, R} \\
& \leqslant 2 A R^{\alpha}+C\left(\frac{r}{R}\right)^{\gamma}\left(2 A R^{\alpha}+\underset{B(x, R)}{\operatorname{osc}} u\right) \leqslant 2 A(C+1) R^{\alpha}+C\left(\frac{r}{R}\right)^{\gamma} \underset{B(x, R)}{\operatorname{Osc}} u,
\end{aligned}
$$

где $A, C \geqslant 0, A$ зависит только от функции $u$ и от $\alpha$, а $C$ - только от $n, \lambda$ и $\Lambda$. Применяя лемму 4 , отсюда заключаем, что при $\alpha<\gamma$ функция $u$ принадлежит классу $C^{\alpha}(G)_{\text {loc }}$.

Теорема доказана.

\section{ЛитерАТУРА}

[1] Е. М. Ландис, Уравнения второго порядка эллиптического и параболического тиnа, Наука, М., 1971.

[2] Д. Гилбарг, Н. Трудингер, Эллиптические дифференииалъные уравнения с частными производными второго порядка, Наука, М., 1989.

[3] M. Grüter, K.-O. Widman, The Green function for uniformly elliptic equations, Manuscripta Math., 37 (1982), 303-342.

[4] Е. П. Долженко, О «стирании» особенностей аналитических функиий, УМН, $\mathbf{1 8 : 4 ~ ( 1 1 2 ) ~ ( 1 9 6 3 ) , ~ 1 3 5 - 1 4 2 . ~}$

[5] L. Carleson, Removable singularities for continuous harmonic functions in $\mathbb{R}^{n}$, Math. Scand., 12 (1963), 15-18.

[6] Е. П. Долженко, О представлении непрерывных гармонических функиий в виде потенциалов, Изв. АН СССР, сер. матем., 28:5 (1964), 1113-1130.

[7] Е. П. Долженко, Об особъх точках непрерывных гармонических функций, Изв. АН СССР, сер. матем., 28:6 (1964), 1251-1270.

[8] Л. Карлесон, Избранные проблемы теории исключительных множеств, Мир, М., 1971.

[9] R. Harvey, J. C. Polking, Removable singularities of solutions of linear partial differential equations, Acta Math., 125:1/2 (1970), 39-56.

[10] J. Kral, Semielliptic singularities, Časopis Pěst. Mat., 109 (1984), 304-322.

[11] Б. Ж. Ищанов, О представлении решений некоторых классов уравнений в виде потенииалов мер, распределенных на множестве особенностей этих решений, Докл. РАН, 358:4 (1998), 455-458. 
[12] J. Mateu, J. Orobitg, Lipschitz approximations by harmonic function, Indiana Univ. Math. J., 39:3 (1990), 703-736.

[13] D. C. Ullich, Removable sets for harmonic functions, Mich. Math. J., 38:3 (1991), 467-473.

[14] Н. Н. Тарханов, Ряд Лорана для решений эллиптических систем, Наука, Новосибирск, 1991.

[15] G. David, P. Mattila, Removable sets for Lipschitz harmonic functions in the plane, Rev. Mat. Iberoamericana, 16 (2000), 137-215.

[16] А. В. Покровский, Локальные аппроксимации решениями гипоэллиптических уравнений и устранимые особенности, Докл. РАН, 367:1 (1999), 15-17.

[17] T. Kilpeläinen, X. Zhong, Removable sets for continuous solutions of quasilinear elliptic equations, Proc. Amer. Math. Soc., 130:6 (2002), 1681-1688 (electronic).

[18] А. В. Покровский, Устранимые особенности р-гармонических функций, Дифференциальные уравнения, 41:7 (2005), 897-907.

[19] А. В. Покровский, Устранимые особенности решений уравнения минималъных поверхностей, Функц. анализ и его прил., 39:4 (2005), 62-68.

[20] В. С. Федоров, Sur la representatione des fonctions analitiques au voisinage d'un ensemble de ses points singuliers, Матем. сб., 35 (1928), 237-250.

[21] А. В. Покровский, Устранимые особенности решений дивергентных эллиптических уравнений второго порядка, Матем. заметки, 77:3 (2005), 424-433.

[22] А. В. Покровский, Классы функций, определяемые с помощъю локалъных приближений решениями гипоэллиптических уравнений, Сиб. матем. журн., 47:2 (2006), 394-413.

[23] В. А. Кондратьев, Е. М. Ландис, Качественная теория линейных дифференииалъных уравнений в частных производных второго порядка, в кн.: Итоги науки и техники. Современные проблемы математики. Фундаментальные направления, т. 32, ВИНИТИ, М., 1988, 99-215.

[24] L. A. Caffarelli, E. B. Fabes, C. E. Kenig, Completeli singular elliptic-harmonic measures, Indiana Univ. Math. J., 30:6 (1981), 917-924.

[25] W. Littman, G. Stampacchia, H. F. Weinberger, Regular points for elliptic equations with discontinuous coefficients, Ann. Scuola Norm. Super. Pisa (III), 17 (1963), 43-77.

[26] M. Giaquinta, Multiple Integrals in the Calculus of Variations and Nonlinear Elliptic Systems, Princeton Univ. Press, Princeton, 1983.

Институт математики НАН Украины

Поступило в редакцию e-mail: pokrovsk@imath.kiev.ua 15 сентября 2006 г. 